\title{
$1 \quad$ MRI and CT imaging biomarkers of cerebral amyloid angiopathy in lobar
}

2 intracerebral haemorrhage

4 Ghil Schwarz ${ }^{1,2}$, Gargi Banerjee ${ }^{1}$, Isabel C. Hostettler ${ }^{1,3}$, Gareth Ambler ${ }^{4}$, David J. Seiffge ${ }^{1,5}$, Hatice

5 Ozkan $^{1}$, Simone Browning ${ }^{1}$, Robert Simister ${ }^{1}$, Duncan Wilson ${ }^{1,6}$, Hannah Cohen ${ }^{7}$, Tarek Yousry ${ }^{8}$,

6 Rustam Al-Shahi Salman ${ }^{9}$, Gregory Y.H. Lip ${ }^{10}$, Martin M. Brown ${ }^{1}$, Keith W. Muir ${ }^{11}$, Henry Houlden ${ }^{12}$,

7 Rolf Jäger $^{8}$, David J. Werring ${ }^{1}$ on behalf of the CROMIS-2 and SIGNaL investigators

$9{ }^{1}$ Stroke Research Centre, University College London, Institute of Neurology, London, UK

$10{ }^{2}$ Department of Neurology and Stroke Unit ASST Grande Ospedale Metropolitano Niguarda, Milan, 11 Italy

$12{ }^{3}$ Department of Neurosurgery, Cantonal Hospital St. Gallen, St. Gallen, Switzerland

$13{ }^{4}$ Department of Statistical Science, University College London, Gower Street, London, UK

$14{ }^{5}$ Department of Neurology and Stroke Center, Inselspital, Bern, Switzerland

$15{ }^{6}$ New Zealand Brain Research Institute, Christchurch, New Zealand

$16{ }^{7}$ Haemostasis Research Unit, Department of Haematology, University College London, 51 Chenies 17 Mews, London, UK

$18{ }^{8}$ Neuroradiological Academic Unit, Department of Brain Repair and Rehabilitation, UCL Institute of 19 Neurology, Queen Square, London, UK and Lysholm Department of Neuroradiology, The National 20 Hospital for Neurology and Neurosurgery, Queen Square London

$21{ }^{9}$ Centre for Clinical Brain Sciences, School of Clinical Sciences, University of Edinburgh, Edinburgh, $22 U K$

$23{ }^{10}$ Liverpool Centre for Cardiovascular Science, University of Liverpool and Liverpool Heart \& Chest 24 Hospital, Liverpool, United Kingdom; and Department of Clinical Medicine, Aalborg University, 25 Aalborg, Denmark

${ }^{11}$ Institute of Neuroscience \& Psychology, University of Glasgow, Queen Elizabeth University Hospital, Glasgow, UK

${ }^{12}$ Department of Molecular Neuroscience, UCL Institute of Neurology and the National Hospital for Neurology and Neurosurgery, Queen Square, London 
31 Corresponding author: Professor David Werring, FRCP, PhD, National Hospital of Neurology

32 and Neurosurgery, Institute of Neurology, University College London, Queen Square, WC1N

33 London, United Kingdom, Phone: +44 203447 5994, Fax: +44 207833 8613, Email:

34 d.werring@ucl.ac.uk

35 Keywords: Lobar intracerebral haemorrhage, cerebral amyloid angiopathy, CAA, modified Boston 36 criteria, full Edinburgh criteria, simplified Edinburgh criteria.

Manuscript word count: 3997

Abstract word count: 256

41 Title characters count (including spaces): 96

Figure count: 1

Table count: 3

References: 19

Sources of funding:

49 GB holds an NIHR Academic Clinical Fellowship, and has received funding from the Rosetrees

50 Trust. DJW receives funding from the Stroke Foundation and British Heart Foundation. RS

51 receives funding from $\mathrm{UCLH} / \mathrm{UCL} B R C$. $\mathrm{HH}$ and $\mathrm{ICH}$ received funding from the Alzheimer

Research UK and Dunhill Medical Trust Foundation. This work was undertaken at UCLH/UCL which receives a proportion of funding from the Department of Health's National Institute for Health Research (NIHR) Biomedical Research Centres funding scheme. The remaining authors declare no financial or other conflicts of interest. 


\section{ABSTRACT}

Background. Cerebral amyloid angiopathy (CAA), a common cause of intracerebral haemorrhage $(\mathrm{ICH})$, is diagnosed using the Boston criteria including MRI biomarkers (cerebral microbleeds [CMB] and cortical superficial siderosis [CSS]). The simplified Edinburgh criteria include CT biomarkers (subarachnoid extension [SAE] and finger-like projections [FLP]). The underlying mechanisms and diagnostic accuracy of CT compared to MRI biomarkers of CAA are unknown.

Methods. We included 140 survivors of spontaneous lobar supratentorial ICH with both acute CT and MRI. We assessed associations between MRI and CT biomarkers and the diagnostic accuracy of CT- compared to MRI-based criteria.

Results. FLP were more common in patients with strictly lobar CMB (44.7\% vs $23.5 \% ; p=0.014)$ and SAE was more common in patients with cSS $(61.3 \%$ vs $31.2 \% ; p=0.002)$. The high probability of the CAA category of the simplified Edinburgh criteria showed $87.2 \%(95 \% \mathrm{Cl} 78.3-93.4)$ specificity, $29.6 \%(95 \% \mathrm{Cl} 18.0-43.6)$ sensitivity, $59.3 \%(95 \% \mathrm{Cl} 38.8-77.6)$ positive predictive value and $66.4 \%$ (95\% Cl 56.9-75.0), negative predictive value, $2.3(95 \% \mathrm{Cl} 1.2-4.6)$ positive likelihood ratio and 0.8 (95\% Cl $0.7-1.0)$ negative likelihood ratio for probable CAA (vs non-probable CAA), defined by the modified Boston criteria; the area under the receiver operating curve (AUROC) was $0.62(95 \% \mathrm{CI}$ 0.54-0.71).

Conclusion. In lobar $\mathrm{ICH}$ survivors, we found associations between putative biomarkers of parenchymal CAA (FLP and strictly lobar CMBs) and putative biomarkers of leptomeningeal CAA (SAE and cSS). CT biomarkers might help rule-in probable CAA (diagnosed using the Boston criteria), but their absence is probably not useful to rule it out, suggesting an important continued role for $\mathrm{MRI}$ in $\mathrm{ICH}$ survivors with suspected CAA. 


\section{INTRODUCTION}

Spontaneous lobar intracerebral haemorrhage $(\mathrm{ICH})$ related to cerebral amyloid angiopathy $(\mathrm{CAA})$ is associated with high risks of death, poor functional outcome, dementia [1] and intracerebral hemorrhage $(\mathrm{ICH})$ recurrence [2], so it is important to identify in clinical practice. Histopathological assessment is the reference standard to identify CAA, but cerebral tissue is rarely available, so neuroimaging biomarkers are usually used to infer the presence of CAA. The modified Boston criteria for CAA [3][4] are widely used MRI-based criteria. However, MRI is not always available, tolerated, or possible due to contraindications, particularly during acute care.

More recently, the acute CT-based Edinburgh criteria have been proposed [5]; a CT-only version of the criteria (the simplified Edinburgh criteria) include only subarachnoid extension (SAE) and fingerlike projections (FLP). The Edinburgh criteria demonstrated excellent diagnostic accuracy for autopsy-proven CAA in severe $\mathrm{ICH}$ (fatal events), but still require external validation. Furthermore, little is known about the underlying mechanisms of FLP or SAE. FLP might reflect CAA affecting brain parenchymal small vessels (causing blood to dissect into abnormal brain tissue), while SAE might be due to leptomeningeal arteriolar CAA (leading to acute bleeding into the subarachnoid space).

We aimed to evaluate: (1) whether FLP are associated with CMBs (as a putative biomarker of parenchymal CAA); (2) whether SAE is associated with cSS (as a putative biomarker of leptomeningeal CAA); and (3) to evaluate the diagnostic accuracy and concordance of simplified Edinburgh criteria compared to modified Boston criteria.

\section{METHODS}

We retrospectively included consecutive adult patients with spontaneous (non-traumatic) ICH from: an observational prospective multicenter cohort study (Clinical Relevance of Microbleeds in Stroke; CROMIS-2 [ICH] - NCT02513316 [6]) and from the SIGNaL register (Stroke InvestiGation in North and Central London). We included patients with $\mathrm{ICH}$ and both $\mathrm{CT}$ and MRI performed after the index event. Exclusion criteria were: age < 55 years; and non-lobar, infratentorial or secondary ICH (Figure 1). We reported the study in accordance with STARD reporting guidelines. [7] [8] Measurement of 
$113 \mathrm{ICH}$ volume was performed on CT scans via a semi-automated approach [9] and on MRI by manual 114 segmentation on SWI sequences. ICH location was assessed using the Cerebral Haemorrhage 115 Anatomical RaTing inStrument (CHARTS) [10].

116 All neuroimaging biomarkers were rated by a single trained rater, blinded to other clinical and 117 neuroradiological data. Observers evaluated CT for FLP and SAE as previously described [5] after 118 attending a web-based training module (www.ed.ac.uk/edinburgh-imaging/ecciting). Each patient 119 was categorized for the probability of CAA using the simplified Edinburgh criteria (with a high 120 probability defined by the presence of both FLP and SAE) [5] In the derivation study [5], no 121 participants had FLP in isolation, but given the strong association between CAA and FLP [5] we 122 classified FLP in isolation as intermediate risk of CAA. To obtain inter-rater reliability a random 123 sample of 19 CT scans (SIGNaL cohort) was rated by a blinded experienced Stroke Neurologist 124 (DJW).

125 CMBs and cSS were rated on T2*-weighted gradient-recalled echo (GRE) or susceptibility-weighted 126 imaging (SWI) using a validated rating scale [11] and per consensus criteria [12][13], respectively. 127 The typical appearance of cSS ("track-like" low signal in the subpial layers of cortex either side of 128 the sulcus) and the distance in space from the symptomatic $\mathrm{ICH}$ were used to distinguish cSS from 129 acute convexity subarachnoid haemorrhage (SAH). No patients with isolated convexity SAH were 130 included. Each patient was categorized using the modified Boston criteria [3]. We compared the 131 probable CAA category to all other lobar ICH (namely, non-probable CAA: including possible CAA 132 and lobar $\mathrm{ICH}$ not meeting the criteria for CAA [i.e. patients with no additional haemorrhagic CAA 133 markers (lobar CMBs or cortical siderosis) or $\geq 1$ deep CMB]). From the CROMIS-2 cohort a random $13410 \%$ sample (149 scans) was rated to quantify intra-rater and inter-rater reliability for CMBs. For cSS 135 presence the entire cohort of patients included in the SIGNaL cohort (42 scans, $30 \%$ of the entire 136 cohort) were rated twice for intra-rater reliability.

137 Univariate analysis was performed to evaluate association between variables and categories; the 138 strength of associations was quantified via agreement proportion and kappa (k) values. The 139 diagnostic accuracy of a high probability of CAA (according to the simplified Edinburgh criteria) in 140 predicting probable CAA (according to the modified Boston criteria) was assessed by calculating the 
141 area under the receiver operating characteristic curve (AUROC), sensitivity, specificity, positive and 142 negative predictive values. Positive and negative likelihood ratios ( $\mathrm{R}_{+}$and $\left.\mathrm{LR}-\right)$ were also 143 calculated. Univariable and multivariable (adjusted for age and sex) linear regression analysis was 144 performed to assess if presence of CT (SAE or FLP) and MRI (strictly lobar CMB or cSS) biomarkers 145 of CAA were correlated with ICH volume. To test for selection bias, we compared (univariate 146 analysis) patients with and without available MRI. Inter/intra-observer variability of ratings was 147 calculated using the Cohen $\mathrm{k}$ statistic. The significance level was set at $p=0.05$. Statistical analysis was performed using STATA 16 (StataCorp. 2019 Stata Statistical Software: Release 16).

Standard Protocol Approvals, Registrations and patient consents. Written informed consent was obtained from all participants in CROMIS-2 (approved by UK National Health Service

152 Research Ethics Committee: 10/H0716/64); in case of lack of capacity written informed consent was 153 obtained from a relative or representative. For the SIGNaL cohort, data were collected as part of 154 routine clinical care and data analysis was approved as a service evaluation by the University College London Hospitals NHS Trust Data Governance Review Board.

Data Availability Statement. All de-identified participant data requests should be submitted to the corresponding author for consideration by the CROMIS-2 and SIGNaL Steering Committees.

\section{RESULTS}

160 We included 140 adult patients with spontaneous lobar supratentorial ICH. Baseline characteristics, 161 neuroimaging variables and classifications according to the Edinburgh and Boston criteria are 162 reported in Table 1.

163 Associations between CT and MRI biomarkers (with agreement proportion and $\mathrm{k}$ values) are 164 reported in Table 2. FLP presence was associated with CMB presence (35.8\% vs 20.3\%; $p=0.047)$, 165 strictly lobar CMBs (44.7\% vs $23.5 \% ; p=0.014)$ and total CMB count $(p=0.013)$. FLP were not 166 significantly more common in patients with cSS (35\% vs $27.5 \% ; p=0.390)$ and were not associated with cSS severity $(p=0.691)$. SAE was more common in patients with cSS $(61.3 \%$ vs $31.2 \%$; 
$168 \mathrm{p}=0.002)$, and was associated with cSS severity $(\mathrm{p}=0.002)$. SAE was not significantly associated 169 with $\mathrm{CMB}$ presence (37\% vs $39 \%$; $\mathrm{p}=0.815)$, strictly lobar CMB $(47.4 \%$ vs $34 \% ; \mathrm{p}=0.157)$ or $\mathrm{CMB}$ 170 count $(p=0.787)$.

171 Compared to patients without probable CAA, FLP were more common in patients with probable CAA $172(40.7 \%$ vs $22.1 \%, p=0.018)$. Compared to patients without probable CAA, SAE was more common 173 in patients with probable CAA ( $51.9 \%$ vs $29.1 \%, p=0.007)$. In both cases the agreement proportion 174 was $63.6 \%(95 \% \mathrm{Cl} 55.0-71.5)$.

175 Adopting probable CAA based on the modified Boston criteria as the diagnostic reference, a high 176 probability of CAA according to the simplified Edinburgh criteria showed specificity $87.2 \%(95 \% \mathrm{Cl}$ 177 78.3-93.4), sensitivity 29.6\% (95\%Cl 18.0-43.6), positive predictive value $59.3 \%(95 \% \mathrm{Cl} 38.8-$ 178 77.6), negative predictive value 66.4\% (95\%Cl 56.9-75.0), LR+ $2.3(95 \% \mathrm{Cl} 1.2-4.6)$ and LR- 0.8 $179(95 \% \mathrm{Cl}$ 0.7-1.0). The discrimination (AUROC) of the simplified Edinburgh criteria (high probability 180 vs intermediate or low probability), for probable CAA according to the Boston criteria (vs non181 probable) was $0.62(95 \% \mathrm{Cl} 0.54-0.71)$ (Table 3).

182 The median ICH volume was significantly higher when FLP were present $(20.4 \mathrm{ml}$ vs $7.7 \mathrm{ml} ; \mathrm{p}$ $183<0.001)$ or SAE (16.7 vs $6.7 \mathrm{ml} ; \mathrm{p}<0.001)$; these differences remained significant after correcting 184 for age and gender $(p<0.001)$. We found no differences in the presence of cSS and strictly lobar $185 \mathrm{CMBs}$ according to $\mathrm{ICH}$ volume. When we assessed the subgroup of patients with $\mathrm{ICH}$ volume 186 greater than the median value of our cohort $(12.0 \mathrm{~mL})$, the sensitivity of Edinburgh criteria increased 187 from $29.6 \%(95 \% \mathrm{Cl} 18.0-43.6)$ to $50.0 \%(95 \% \mathrm{Cl} 27.2-72.9)$, while specificity was slightly reduced 188 at $77.4 \%(95 \% \mathrm{Cl} 58.9-90.4)$.

189 Comparison between patients with and without available MRI (Table e1) and intra/inter-rater 190 reliability for the presence of CAA biomarkers (Table e2) are reported in Supplementary material.

\section{DISCUSSION}

194 In this study of patients with spontaneous lobar ICH we found significant and specific associations 195 between FLPs and SAE (on CT) and CMBs and CSS (on MRI), respectively. These observations 
provide new insights into the mechanisms and anatomical distribution of the underlying CAA pathology: FLPs are likely to represent parenchymal-predominant CAA (indicated by strictly lobar CMBs on MRI), while SAE might reflect leptomeningeal-predominant CAA (indicated by cSS on $\mathrm{MRI})$. We also found that the prevalence of CT biomarkers increased with the degree of diagnostic certainty regarding CAA defined by the modified Boston criteria and with the volume of $\mathrm{ICH}$. CT diagnostic biomarkers for CAA could be useful in everyday clinical practice, but have only been validated in patients who suffered fatal $\mathrm{ICH}$ [5]. Our study in $\mathrm{ICH}$ survivors showed that the Edinburgh CT-only criteria [5] do increase the likelihood of CAA (defined by the Boston MRI-based criteria), but to a modest extent $(\mathrm{LR}+2.3[95 \% \mathrm{Cl} 1.2-4.6]$; a $\mathrm{LR}+$ of more than 3 is considered to be a good test to rule in a disease). Nevertheless, when a diagnosis of CAA is suspected and MRI is not available (i.e. very unwell or claustrophobic patients, non-MRI compatible implanted devices), the presence of both SAE and FLP on CT might help to rule-in CAA but their absence is probably not useful to rule it out (LR- $0.8[95 \% \mathrm{Cl} 0.7-1.0]$; a LR- of less than 0.33 is considered a good test to rule out a disease).

The original Edinburgh criteria validation study [5] found that all cases with high or intermediate probability of having moderate or severe CAA were classified as probable CAA by the Boston criteria, but this analysis was available for only 7 patients (with both CT and MRI available). A recent study [14] found that FLP presence (on CT) was significantly more frequent in probable than in possible CAA, but did not specifically examine associations between CT and MRI biomarkers. Our findings are consistent with these previous observations, but also provide new evidence regarding the underlying mechanisms and diagnostic accuracy of the simplified Edinburgh acute CT criteria. Another recent study [15] on Dutch-type hereditary CAA patients documented that the presence of FLP and SAE correlate with ICH volume with higher sensitivity of simplified Edinburgh criteria in large $\mathrm{ICH}$ volumes. Our results are in line with this finding: when simplified Edinburgh criteria were applied in patients with $\mathrm{ICH}$ volume greater than $12 \mathrm{~mL}$ (the median volume), sensitivity increased with only slightly lower specificity. Further studies may be helpful to determine whether a minimum 
$223 \mathrm{ICH}$ volume cutoff point should be considered to maximize the diagnostic accuracy of the Edinburgh 224 criteria.

225 CAA is not a uniform disease, having a complex range of clinical, imaging and neuropathological subtypes [16]. An autopsy-based study described two CAA phenotypes [17]: in CAA type 1, amyloid 227 beta-protein (A-beta) is primarily found in cortical capillaries, while in CAA type 2 A-beta is primarily 228 deposited in leptomeningeal and cortical vessel, sparing cortical capillaries. These phenotypes are 229 hypothesized to be partially driven by APOE genotype: APOE e4 is associated with type 1 230 (parenchymal-predominant) CAA, while APOE e2 is associated with type 2 (leptomeningeal231 predominant) CAA [17]. In line with these recent histopathological observations, two recent meta232 analyses found that strictly lobar CMB are related to APOE e4 [18] and that cSS is most strongly 233 associated with APOE e2 genotype [19]. We found strong association between cSS and SAE 234 presence, and between CMBs (especially strictly lobar CMBs) and FLP presence. Our results 235 suggest that FLP and SAE might be related to different anatomical distributions of CAA pathology, 236 which may in part be related to underlying APOE genotype.

237 Our study has strengths. We included a consecutive sample of participants with lobar ICH. CT and 238 MRI were assessed by trained blinded experienced raters with standardized rating instruments and 239 consensus criteria with substantial or excellent intra-rater and inter-rater agreement. Moreover, MRI 240 scans were performed soon after CT; for patients included in the SIGNaL cohort the median was 2 241 days (IQR 1-3).

242 We also acknowledge limitations. The requirement of an MRI scan and of signed informed consent 243 could have created a selection bias towards non-severe, clinically stable ICH patients. The patients 244 with MRI available were significantly younger, but there was not a major difference in clinical severity. 245 We could not evaluate the accuracy of Edinburgh criteria against histopathological assessment, 246 which is the reference standard for a diagnosis of CAA. However, histopathological analysis of brain 247 tissue is rarely performed in clinical practice, while in clinical practice the diagnosis of CAA is often 248 made based on the modified Boston criteria, which show good diagnostic accuracy for 249 pathologically-proven CAA in ICH (specificity $81.2 \%$ [95\% Cl 61.5-92.7], sensitivity 94.7\% [95\% Cl 250 82.7-98.5]) [3]. While our findings need to be validated against histopathological assessment, they 
251 remain relevant to guide clinicians in every day clinical practice, especially where MRI is not 252 available.

\section{CONCLUSION}

254 We have shown associations between putative biomarkers of parenchymal CAA (FLP and CMB), 255 and between putative biomarkers of leptomeningeal CAA (SAE and cSS). Our findings indicate that, 256 in lobar ICH survivors where CAA is suspected, CT biomarkers suggesting a high probability of CAA 257 might help rule-in MRI-defined probable CAA. However, the absence of FLP and SAE on CT are 258 probably not useful to rule-out the presence of CAA, suggesting an important continued role for MRI 259 in the investigation of ICH survivors with suspected CAA. 


\section{REFERENCES}

274 [1] Z Arvanitakis et al., The Relationship of Cerebral Vessel Pathology to Brain Microinfarcts. Brain 275 Pathol. 2017 Jan;27(1):77-85.

276 [2] A Charidimou, et al. Brain hemorrhage recurrence, small vessel disease type, and cerebral 277 microbleeds, Neurology, vol. 89, no. 8, pp. 820-829, Aug. 2017.

278 [3] J Linn, et al. Prevalence of superficial siderosis in patients with cerebral amyloid angiopathy, pp. 279 1-6, Apr. 2010.

280 [4] SM Greenberg, et al. Diagnosis of Cerebral Amyloid Angiopathy Evolution of the Boston Criteria, 281 pp. 1-7, Jan. 2018.

282 [5] MA Rodrigues, et al. The Edinburgh CT and genetic diagnostic criteria for lobar intracerebral 283 haemorrhage associated with cerebral amyloid angiopathy: model development and diagnostic test 284 accuracy study, The Lancet Neurology, vol. 17, no. 3, pp. 232-240, Feb. 2018.

285 [6] A. Charidimou, et al. The Clinical Relevance of Microbleeds in Stroke study (CROMIS-2): 286 rationale, design, and methods, International Journal of Stroke, vol. 10, no. 100, pp. 155-161, Oct. 2872015.

288 [7] PM Bossuyt, et al. STARD 2015: an updated list of essential items for reporting diagnostic 289 accuracy studies, pp. 1-9, Oct. 2015.

290 [8] Cohen JF, et al STARD for Abstracts: Essential items for reporting diagnostic accuracy studies 291 in journal or conference abstracts. BMJ 2017;358:j3751

292 [9] B Volbers, et al. Semi-automatic volumetric assessment of perihemorrhagic edema with computed 293 tomography, Eur J Neurol, vol. 18, no. 11, pp. 1323-1328, Apr. 2011. 
294 [10] A Charidimou, et al. The Cerebral Haemorrhage Anatomical RaTing inStrument (CHARTS): 295 Development and assessment of reliability, Journal of the Neurological Sciences, vol. 372, no. C, 296 pp. 178-183, Jan. 2017.

297 [11] SM Gregoire, et al. The Microbleed Anatomical Rating Scale (MARS), pp. 1-9, Nov. 2009.

298 [12] A Charidimou, et al. Cortical superficial siderosis: detection and clinical significance in cerebral 299 amyloid angiopathy and related conditions, Brain, vol. 138, no. 8, pp. 2126-2139, Jul. 2015.

300 [13] JM Wardlaw, et al. Neuroimaging standards for research into small vessel disease and its 301 contribution to ageing and neurodegeneration, The Lancet Neurology, vol. 12, no. 8, pp. 822-838, 302 Jul. 2013.

303 [14] D Renard, et al. Finger-Like Projections in Lobar Haemorrhage on Early Magnetic Resonance 304 Imaging Is Associated with Probable Cerebral Amyloid Angiopathy., Cerebrovasc Dis, vol. 47, no. 3, 305 pp. 121-126, 2019.

306 [15] ES V Etten, et al. Sensitivity of the Edinburgh Criteria for Lobar Intracerebral Hemorrhage in 307 Hereditary Cerebral Amyloid Angiopathy. Stroke. 2020 Dec;51 (12):3608-3612. 308

309 [16] Charidimou A, et al. Emerging concepts in sporadic cerebral amyloid angiopathy. Brain. 2017 310 Jul 1;140(7):1829-1850.

311 [17] DR Thal, et al. Two Types of Sporadic Cerebral Amyloid Angiopathy, pp. 1-12, Feb. 2002.

312 [18] S Schilling, et al. APOE genotype and MRI markers of cerebrovascular disease, pp. 1-9, Nov. 313 1BC.

314 [19] A Charidimou, et al. APOE and cortical superficial siderosis in CAA: Meta-analysis and potential 315 mechanisms. Neurology, vol. 93, no. 4, pp. e358-e371, Jul. 2019. 
Table 1. General characteristics of the cohort

\begin{tabular}{|c|c|}
\hline Clinical variables & $\mathbf{N}(\%)$ \\
\hline Age (median; IQR) & $72.5(65-78)$ \\
\hline Female gender & $81(57.9)$ \\
\hline Hypertension & $82(58.6)$ \\
\hline Oral anticoagulant drug at index $\mathrm{ICH}$ & $28(20.0)$ \\
\hline Prior ICH & $14(10.0)$ \\
\hline Glasgow Coma Scale at admission (median; IQR) & $15(1)$ \\
\hline $\mathrm{ICH}$ volume (median [IQR]) ${ }^{\#}$ & $12.0(4.5-20.0)$ \\
\hline MRI-based variables and criteria & $\mathbf{N}(\%)$ \\
\hline \multicolumn{2}{|l|}{ Cerebral microbleed } \\
\hline Absent & $59(42.1)$ \\
\hline Present & $81(57.9)$ \\
\hline $1-5$ & $37(26.4)$ \\
\hline $6-10$ & $14(10.0)$ \\
\hline $11-20$ & $14(10.0)$ \\
\hline$>20$ & $16(11.4)$ \\
\hline Lobar CMB presence & $63(45.0)$ \\
\hline Strictly lobar CMB presence & $38(27.1)$ \\
\hline Deep CMB presence & $31(22.1)$ \\
\hline Brainstem CMB presence & $16(11.4)$ \\
\hline Infratentorial CMB presence & $39(27.9)$ \\
\hline \multicolumn{2}{|l|}{ Cortical superficial siderosis } \\
\hline Absent & 109 (77.9) \\
\hline Present & $31(22.1)$ \\
\hline Focal & $17(12.1)$ \\
\hline Disseminated & $14(10.0)$ \\
\hline \multicolumn{2}{|l|}{ Modified Boston criteria } \\
\hline Non-probable CAA & $86(61.4)$ \\
\hline Probable CAA & $54(38.6)$ \\
\hline
\end{tabular}

CT-based variables and criteria

$\begin{array}{cc}\text { Finger-like projection presence } & 41(29.3) \\ \text { Subarachnoid extension presence } & 53(37.9)\end{array}$

Simplified Edinburgh criteria

Low probability of CAA $73(52.1)$

Intermediate probability of CAA $40(28.6)$

High probability of CAA $27(19.3)$

$I Q R$, Interquartile range; ICH, intracerebral haemorrhage; $C M B$, cerebral microbleeds; $C A A$, cerebral amyloid angiopathy

\# Volume in $\mathrm{mL}$; data available for 101 patients (72\% of the entire cohort). 
Table 2.

Association between FLP and MRI biomarkers

Finger-like projections

$$
\begin{array}{ccc}
P \text { value } & \begin{array}{c}
\text { Agreement } \% \\
(95 \% \mathrm{Cl})
\end{array} & \kappa \text { value } \\
& (95 \% \mathrm{Cl})
\end{array}
$$

\section{CMB}

\section{Absent Present}

$0.047^{\star} \quad 54.3(45.7-62.7)$

$0.142(0.006-$

$0.277)$

Absence $47(79.7) \quad 12(20.3)$

Strictly Lobar CMB

$52(64.2)$

$29(35.8)$

$\begin{array}{rcr}\text { No } & 78(76.5) & 24(23.5) \\ \text { Yes } & 21(55.3) & 17(44.7) \\ \text { CMB count } & & \\ 0 & 47(79.7) & 12(20.3) \\ 0-5 & 28(75.7) & 9(24.3) \\ 6-10 & 5(35.7) & 9(64.3) \\ 11-20 & 11(78.6) & 3(21.4) \\ >20 & 8(50.0) & 8(50.0) \\ \text { cSS } & & \\ & & \\ \text { Absent } & 79(72.5) & 30(27.5) \\ \text { Present } & 20(64.5) & 11(35.5) \\ \text { cSS severity } & & \\ \text { Absent } & 79(72.5) & 30(27.5) \\ \text { Focal } & 11(64.7) & 6(35.3) \\ \text { Disseminated } & 9(64.3) & 5(35.7)\end{array}$

$0.013 \S$

$0.390^{*}$

$64.3(55.8-72.2)$

$0.071(-0.097-$

\begin{tabular}{|c|c|c|c|c|c|}
\hline & \multicolumn{2}{|c|}{ Subarachnoid extension } & \multirow[t]{2}{*}{$P$ value } & \multirow{2}{*}{$\begin{array}{c}\text { Agreement \% (95\% } \\
\mathrm{Cl})\end{array}$} & \multirow{2}{*}{$\begin{array}{l}\text { k values } \\
(95 \% C l)\end{array}$} \\
\hline & Absent & Present & & & \\
\hline CMB & & & $0.815^{*}$ & $47.1(38.7-55.8)$ & $\begin{array}{c}-0.018(-0.0171- \\
0.135)\end{array}$ \\
\hline Absence & $36(61.0)$ & $23(39.0)$ & & & \\
\hline Presence & $51(63.0)$ & $29(37.0)$ & & & \\
\hline Strictly Lobar CMB & & & $0.157^{\star}$ & $60.7(52.1-68.9)$ & $\begin{array}{c}0.116(-0.048- \\
0.280)\end{array}$ \\
\hline No & $67(65.7)$ & 35 (34.3) & & & \\
\hline Yes & $20(52.6)$ & $18(47.4)$ & & & \\
\hline CMB count & & & $0.787 \S$ & - & - \\
\hline 0 & $36(61.0)$ & $23(39.0)$ & & & \\
\hline $0-5$ & $23(62.2)$ & $14(37.8)$ & & & \\
\hline $6-10$ & $7(50.0)$ & $7(50.0)$ & & & \\
\hline $11-20$ & $10(71.4)$ & $4(28.6)$ & & & \\
\hline$>20$ & $11(68.8)$ & $5(31.2)$ & & & \\
\hline cSS presence & & & $0.002^{*}$ & $67.1(58.7-74.8)$ & $\begin{array}{c}0.240(0.081- \\
0.399)\end{array}$ \\
\hline Absent & $75(68.8)$ & $34(31.2)$ & & & \\
\hline Present & $12(38.7)$ & $19(61.3)$ & & & \\
\hline cSS severity & & & $0.002^{*}$ & - & - \\
\hline Absent & $75(68.8)$ & $34(31.2)$ & & & \\
\hline
\end{tabular}
$0.240)$

Association between SAE and MRI biomarkers 
Focal $\quad 9(52.9) \quad 8(47.1)$

Disseminated $3(21.4) \quad 11$ (78.6)

FLP, finger-like projections; cSS, cortical superficial siderosis; $C l$, confidence interval CMB, cerebral microbleeds; SAE, subarachnoid extension; * $\mathrm{X}^{2}$ test; \$Wilkoxon rank sum test 
Table 3. Comparison between simplified Edinburgh criteria and modified Boston criteria and discrimination of simplified Edinburgh criteria for Probable CAA (per MRI-based modified Boston criteria)

Classification per simplified Edinburgh criteria and modified Boston criteria: AUC $=0.62(95 \% \mathrm{Cl}$ 0.54-0.71)

Simplified Edinburgh criteria

High probability of CAA Intermediate probability of CAA

Low probability of CAA

Modified Boston criteria

Probable CAA Non-probable CAA

$16(59.3)$

$18(45.0)$

$20(27.4)$
$11(40.7)$

$22(55.0)$

$53(72.6)$
$27(100)$

$40(100)$

$73(100)$

Discrimination, sensitivity, specificity, PPV and NPV of high probability of CAA (vs Intermediate/low probability) for probable CAA (per MRI-based modified Boston criteria)

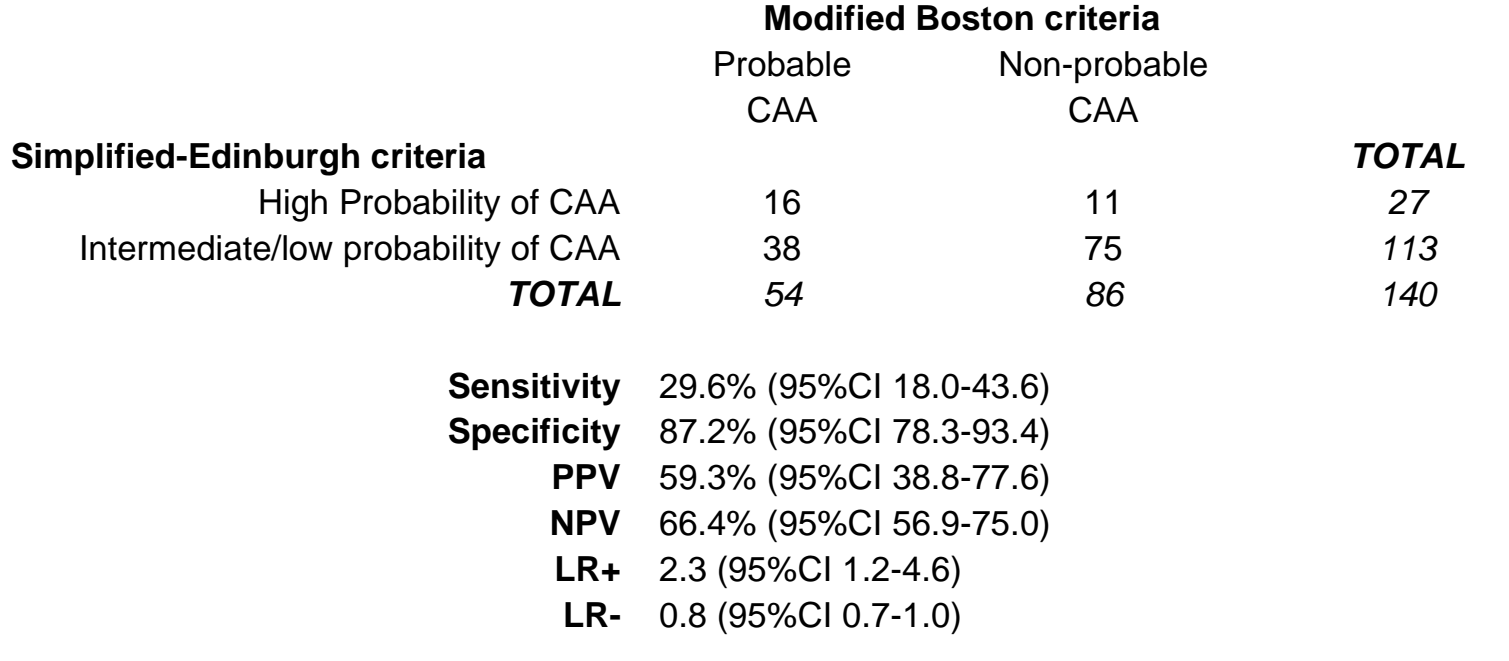

CAA, cerebral amyloid angiopathy; PPV, positive predictive value; $N P V$, negative predictive value. $L R+$, positive likelihood ratio; $L R$-, negative likelihood ratio. 